\title{
Application of two-dimensional finite volume method to protoplanetary disks
}

\author{
Tarik Chakkour ${ }^{1,2}$ \\ ${ }^{1}$ LGPM, CentraleSupélec, Université Paris-Saclay, \\ Centre Européen de Biotechnologie et de Bioéconomie (CEBB), \\ 3 Rue des Rouges-Terres, 51110 Pomacle, \\ France, \\ tarik.chakkour@centralesupelec.fr \\ ${ }^{2}$ LAM, Laboratoire d'Astrophysique de Marseille, \\ 38 Rue Frédéric Joliot Curie, 13013 Marseille, \\ France
}

Received: May 1, 2021. Revised: September 22, 2021. Accepted: October 15, 2021. Published: October $20,2021$.

\begin{abstract}
Many fascinating astrophysical phenomena can be simulated insufficiently by standard numerical schemes for the compressible hydrodynamics equations. In the present work, a high performant 2D hydrodynamical code has been developed. The model is designed for the planetary formation that consists of momentum, continuity and energy equations. Since the two-phase model seems to be hardly executed, we will show in a simplified form, the implementation of this model in one-phase. It is applied to the Solar System that such stars can form planets. The finite volume method (FVM) is used in this model. We aim to develop a first-order well-balanced scheme for the Euler equations in the the radial direction, combined with second-order centered flux following the radial direction. This conception is devoted to balance the fluxes, and guarantee hydrostatic equilibrium preserving. Then the model is used on simplified examples in order to show its capability to maintain steady-state solutions with a good precision. Additionally, we demonstrate the performance of the numerical code through simulations. In particularly, the time evolution of gas orbited around the star, and some properties of the Rossby wave instability are analyzed. The resulting scheme shows consequently that this model is robust and simple enough to be easily implemented.
\end{abstract}

Keywords- Hydrodynamics; protoplanetary disks; finite volume scheme.

\section{INTRODUCTION}

$\mathbf{T}$ HE Planetesimal formation is a complex problem due to great incomprehension process, in where stars of the Solar System form planets. Until today, this is still a matter of debate, because it may eventually give birth to planets in certain variety of conditions that are not very well known yet. The first planets observed outside the Solar System, indicated that planetesimal formation is also possible around massive stars. Stars, like our Sun, takes approximately 1 million years to form, with a protoplanetary disk that will evolve into a planetary system takes from 10 to 100 million years to form. Many observations show this ample evidence. In particularly, the paper [1] shows the detection of two of the most eccentric known planets. In addition, Wang et al. (2006) investigate in [2] fallback disks around young neutron stars.

The Planet formation is possibly occured in the disks around young stars. It is happened in the Solar System where it is considered as a natural result of star formation. Many stars, however, is constitued in multiple stellar system, where the existence of a close neighbour could influence the construction of the disk. The authors investigate in [3] the interaction between particles resulting in star system with different gaseous disks. It has been difficult to show this due to the lack of resolution (interferometric observations). It is concluded that the presence of neighbour perhaps interferes with the creation of planets.

Our study was interested by authors of paper [4], who investigated the evolution of the Rossby wave instability in thin disks using hydrodynamic simulations. They based on the knowledge from the linear theory analysis. They found that this wave instability is robust, purely hydrodynamic mechanism Thus, understanding its appli- 
cations in many astrophysical topics is very important. Here, we report on the results of new $2 \mathrm{D}$ simulations of unstable protoplanetary disk which is extended from 5 to $10 \mathrm{AU}$. Noticing that each astronomical unit $1 \mathrm{AU}$ is equivalent to $1.5 \times 10^{11}$ meters, i.e. $1 \mathrm{AU}=1.5 \times 10^{11} \mathrm{~m}$. They studied the keplerian rotation in the Solar System, in where each solar mass star is shown with its own point.

The numerical methods are important in order to study a lot of problems coming from a wide range of domains, such as astrophysical fluid dynamics. Each field needs algorithms, and we usually look for developing them efficiently. Developing robust algorithms implies its progress. This article investigates a new numerical code for the Planetesimal formation. This code models the flow of particles in disks with refined mesh. Here, it will be comfortably extensible from bidimensional space to three-dimensional one. It combines Godunov method for evaluating the radial flux and the azimuthal flux which is approached by a cell-centred scheme.

Well-balanced finite-volume methods, which preserve a certain class of steady states are explored in many works $[29,30,31,23]$. In this paper, we propose a new class of scheme based on the simplicity and constructions criterions. In order to manage easily the numerical scheme in bidimensional space, our framework consists in balancing the fluxes in each direction. By way of explanation, the main idea is to employ radial numerical flux approached by the Riemann problem solutions with wellbalanced scheme. It induces a conservative flux in the following direction. Next, adjoining an azimuthal flux which is computed by cell-centered scheme. The contribution of this work, is to equilibrate flux component in each direction, and consequently in both diretions without much complications.

In this paper, we are motivated in the creation of $2 \mathrm{D} / 3 \mathrm{D}$ vortices and in their evolution over time scale. This paper is structured as follows. In section III., we compactly characterize the Euler and continuity equations for the motion of gas then take the explicit form. Next, we focus on detailing the used numerical method, with giving the full discretization of the physical domain. These details are found in section IV. that are shared in three subsections. We describe in first subsection $\mathrm{A}$. the $2 \mathrm{D}$ cylindrical domain where we integrate these equations over it. The continuous spatial coordinates $(r, \theta)$ are discretized into a finite size $\left(n_{r}, n_{\theta}\right)$ in each direction, respectively. It is followed by the initial conditions presented in second subsection B.. The concept key of the used finite volume scheme is to conserve variables and quantities over control volume. We introduce these properties in third subsection C.. Next, in section V., we test the performance of the code with simulating the progression of a protoplanetary disk in time. Section VI. deals with stability of the planet formation. We particularly check in this step the unsteady state with perturbing the disk. Finally, section VII. focuses on schematics of the 3D numerical scheme to model the toroidal domain.

\section{Modeling A KePlerian FLOW IN TWO-PHASE}

This section is devoted to examining the formation of planets in a Keplerian disk. We then detail this study in the presence of two-phase. We discuss here the technical details of the modeling the equilibrium over the Keplerian disc. This modeling is presented in Fig. 1. The numerical simulation of two-phase flow is the object of great attention from researchers. For this reason, we talk about it over this section, but we present numerical results showing only a homogeneous single-phase model. Thus, the originality of this work is to present it in a simplified form to be validated numerically, because implement it directly in two-phase seems to be complicated.

Coming back to two-phase flow for following this literature. This interest is due to a lot of applications. We model the flow by a coupled system of Euler equations. This allows to establish practical results, as well as the study of numerical scheme. Two-phase flow is divided into two categories. The first one is to separate flow phase, which is the type of flow that occurs when the debit of gas and liquid are low. The second one is dispersed flow, for example the formation of air bubbles during the agitation of a water bottle.

We define a control volume by a delimited domain of each cell in order to follow the evolution of quantities which is averaged over it. This Eulerian approach allows to optimize the computation time by taking into account the fact that the dispersed phase affects the gas phase. Consequently, for modeling two phases with using the control volume, each these two volumes are separated by an interface. It is through this interface that there has been an interaction between these two phases.

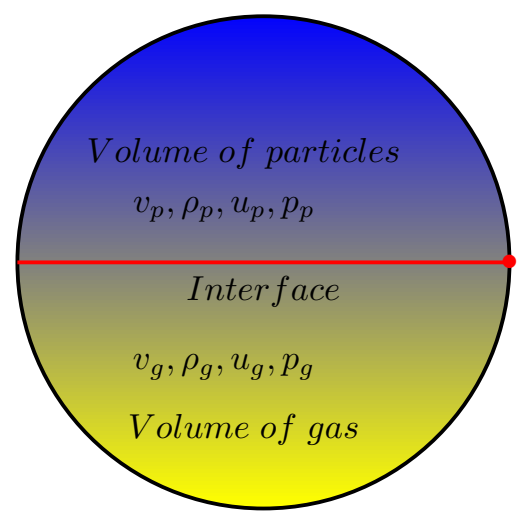

Fig. 1: Modeling of two-phase flow.

Assuming that the disk is at hydrostatic equilibrium along the z-axis, which occurs if the movements along this axis are subsonic. The equilibrium hydrostatic equation involving the pressure $p$ is given by:

$$
\overrightarrow{\operatorname{grad}(p)}=-\rho \cdot \overrightarrow{\operatorname{grad}(\Phi)},
$$

where $\Phi$ is the gravitational potential. If $r \in\left[r_{\min }, r_{\max }\right]$, then the effect of the gravitational field component coming from the mass of disk (self-gravitation) is neglected. This potential $\Phi$ is then written as: $\Phi=-\frac{G M}{D}$, where 
$D=\sqrt{r^{2}+z^{2}}$ is the distance from the considered point of system center. Here, $M$ is the total mass of dust grains falling into the Sun, $D$ is its radius in which $r$ is the radius in the circular orbit and $G$ is the gravitational constant. Consequently, $\Phi$ can be determined over the z-axis by the following equality:

$$
\frac{1}{\rho} \frac{\partial p}{\partial z}=\frac{\partial}{\partial z}\left(\frac{G M}{\sqrt{r^{2}+z^{2}}}\right) .
$$

Assuming that the orbits are circular and obey Kepler's laws. If the disk is thin $z^{2} \ll r^{2}$, we get:

$$
\begin{aligned}
\frac{1}{\rho} \frac{\partial p}{\partial z} & =\frac{\partial}{\partial z} \frac{G M}{r}\left(1-\frac{z^{2}}{2 r^{2}}\right) \\
& =-\frac{G M}{r^{3}} z \\
& =-\Omega^{2} z
\end{aligned}
$$

where $\Omega$ means the angular velocity which is given by:

$$
\Omega=\sqrt{\frac{G M}{r^{3}}} .
$$

The equilibrium over the Keplerian disc allows to give the velocity $v$ in the circular motion as:

$$
v=\sqrt{\frac{G M}{r}} .
$$

The solid particles perform quasi-Keplerian orbits in the plane of the disc, whereas they perform oscillations around the middle equatorial plane in a perpendicular direction at the plane of the disk.

\section{EULER EQUATIONS IN ONE/TWO-PHASE}

The Euler equations [5] express the conservation of three quantities that are the mass, momentum and energy. This conservation is viewed locally as an application of three densities. The first one is velocity shared into two components which are the radial velocity $u$ and the azimuthal velocity $v$. Noticing that the kinetic energy $k$ is the half sum of components velocity squared, i.e. $k=\frac{u^{2}+v^{2}}{2}$. The second one is the pressure denoted by $p$. The last one is the internal energy $E$. Adding the kinetic energy $k$ to the internal one $E$, gives the total energy $e$. Let us consider initially an ideal fluid. For polytropic gas law, with $\gamma>1$, energy, pressure and temperature are given by the following relations:

$$
\left\{\begin{array}{l}
e=k+E \\
p=(\gamma-1) \rho E \\
T=\frac{\mu}{k b} \frac{p}{\rho}
\end{array}\right.
$$

Denoting by $w$, the vector of the conservative variables. The vector $w$ components in cylindrical coordinates are given by:

$$
w=\left(\begin{array}{c}
\rho \\
\rho u \\
\rho v \\
\rho e
\end{array}\right)
$$

The conservation laws involving the cartesian coordinates are written in a one-dimensional space:

$$
\partial_{t} w+\nabla \cdot F(w)=Q(w)
$$

The standard cartesian coordinates $(x, y)$ are given in term of cylindrical coordinates $r$ and $\theta$ :

$$
\left\{\begin{array}{l}
x=r \cdot \cos (\theta) \\
y=r \cdot \sin (\theta) \\
r^{2}=x^{2}+y^{2} \\
\tan (\theta)=\frac{y}{x}
\end{array}\right.
$$

The divergence term $\nabla . F$ in cylindrical coordinates is defined as:

$$
\nabla \cdot F(w)=\frac{\partial F}{\partial x}+\frac{\partial F}{\partial y} .
$$

The partial derivatives of function $F$ with respect to cartesian variables are:

$$
\left\{\begin{array}{l}
\frac{\partial F}{\partial x}=\frac{\partial F}{\partial r} \times \frac{\partial r}{\partial x} \\
\frac{\partial F}{\partial y}=\frac{\partial F}{\partial r} \times \frac{\partial r}{\partial y}
\end{array}\right.
$$

From this, the divergence term $\nabla . F$ is:

$$
\nabla \cdot F(w)=\frac{1}{r} \frac{\partial r F(w)}{\partial r}
$$

The Euler equation is expressed in the radial direction:

$$
\frac{\partial w}{\partial t}+\frac{1}{r} \frac{\partial r F(w)}{\partial r}=Q(w)
$$

The extension of equation (10) to bidimensional cylindrical coordinates is:

$$
\frac{\partial w}{\partial t}+\frac{1}{r} \frac{\partial r F(w)}{\partial r}+\frac{1}{r} \frac{\partial G(w)}{\partial \theta}=Q(w) .
$$

The vector $w$ is written as an application:

$$
w: \mid \begin{aligned}
{\left[0, \infty\left[\times \mathbf{R}_{+}^{\star} \times[0,2 \pi]\right.\right.} & \longrightarrow \mathbf{R}^{\mathbf{3}} \\
(t, r, \theta) & \longmapsto w(t, r, \theta) .
\end{aligned}
$$

The vectors field $F$ and $G$ present respectively the fluxes in the radial and azimuthal directions, defined by the following components:

$$
F(w)=\left(\begin{array}{c}
\rho u \\
\rho u^{2}+p \\
\rho u v \\
(\rho e+p) u
\end{array}\right)
$$

and

$$
G(w)=\left(\begin{array}{c}
\rho v \\
\rho u v \\
\rho v^{2}+p \\
(\rho e+p) v
\end{array}\right),
$$

and where the source term is defined as: 


$$
Q(w)=\left(\begin{array}{c}
0 \\
\frac{\rho v^{2}}{r}-\rho \frac{G M}{r^{2}}+\frac{p}{r}+F_{r} \\
-\frac{\rho u v}{r}+F_{\theta} \\
-\rho u \frac{G M}{r^{2}}
\end{array}\right) .
$$

In which $F_{r}$ and $F_{\theta}$, stand respectively the friction forces in the radial and azimuthal direction, exerted by the gas on the particles per volume unit.

Now, we will determine the explicit analytic representation of the steady-state solutions of equation (10). Indeed, there are two ways to give them. The first one consists to give the stationary solutions in the radial direction independently in the azimuthal one. We check these solutions when the radial velocity is null, i.e. $u_{s t}=0$. Formally,

$$
\frac{\partial r p}{\partial r}=\rho v^{2}-\rho \frac{G M}{r}+p .
$$

Remarking that equation (12) states the hydrostatic equilibrium expressed in cartesian coordinates in section II.. Assuming that temperature $T_{s t}$ and density $\rho_{s t}$ are only given in power of the radius $r$ :

$$
T_{s t}=c_{T} \cdot r^{-\alpha}, \quad \rho_{s t}=c_{\rho} \cdot r^{-\beta} .
$$

In which, $c_{T}$ and $c_{\rho}$ are the real constants. According to the kinetic theory, the state law of a perfect gas (monoatomic) is written as $\rho=\frac{n \mathcal{M}}{\mathcal{N}_{\mathcal{A}}}$, where $n$ and $\mathcal{M}$ design respectively the amount of molecules per unit volume and the molar mass. Then, the pressure $p_{s t}$ is a linear function of product term $T_{s t} \rho_{s t}$ :

$$
p_{s t}=\frac{k_{B}}{\mu} T_{s t} \rho_{s t}
$$

Injecting the expression of $\rho$ and $T_{\text {st }}$ given by (13) in equality (14), we get:

$$
p_{s t}=\frac{c_{T} c_{\rho} k_{B}}{\mu} r^{-\phi}
$$

where $\phi$ is:

$$
\phi=\alpha+\beta \text {. }
$$

Multiplying the stationary pressure $p_{\text {st }}$ resulting from (15) by $r$. Then, taking the derivative again with respect to the same variable. Next, the obtained variable is deducted from this pressure $p_{s t}$ to give:

$$
\frac{\partial r p_{s t}}{\partial r}-p_{s t}=-\phi p_{s t}
$$

Equality (17) is coupled with equation (12) to yield the stationary azimuthal velocity $v_{s t}$ in term of radius $r$ :

$$
v_{s t}=\sqrt{\frac{G M}{r}-\frac{\phi c_{T} k b}{\mu} r^{-\alpha}} .
$$

The derivative of the azimuthal velocity $v_{s t}$ with respect to $r$ :

$$
\frac{\partial v_{s t}}{\partial r}=\frac{-\frac{G M}{r}+\frac{\phi c_{T} k b \alpha}{\mu} r^{-\alpha}}{2 r \sqrt{\frac{G M}{r}-\frac{\phi c_{T} k b}{\mu} r^{-\alpha}}} .
$$

Describing the behavior of velocity $v_{s t}$ in term of the variable $r$. For that, if the power $\alpha$ is supposed vary strictly between the inferior value 0 and the superior value 1 , i.e. $0<\alpha<1$. Then, this velocity $v_{s t}$ is strictly decreasing, expecting to take the maximum velocity at $r_{\min }$, and the minimum velocity at $r_{\max }$. Formally,

$$
\sqrt{\frac{G M}{r_{\max }}-\frac{\phi c_{T} k b}{\mu r_{\max }^{\alpha}}}<v_{s t}<\sqrt{\frac{G M}{r_{\min }}-\frac{\phi c_{T} k b}{\mu r_{\min }^{\alpha}}} .
$$

In what follows, we describe the basic equations of a flow of gas and particles around a star. The protoplanetary disk is detailed in two phase flow, with presence of these particles. Treating the two-phase flows consists in solving simultaneously two conservation equations system. The authors in $[6,7]$ deal with averaging techniques to solve them, even in reality, it is complicated to get numerically solutions. Noticing that we have 9 classical equations, much than some existing models described in $[8,9]$. In order to simplify this model, we neglect the viscosity effect, and thermal diffusion. An other assumption is made, in the sense that solid components effect no pressure on gas. These terms are not involved in the governing equations of this model. The vector of conserved variables $w$, tensor fluxes $F$ and $G$ are defined as:

$$
\begin{gathered}
w=\left(\begin{array}{c}
\rho_{g} \\
\rho u_{g} \\
\rho v_{g} \\
\rho e_{g} \\
\rho_{p} \\
\rho u_{p} \\
\rho v_{p} \\
\rho e_{p} \\
N_{p}
\end{array}\right), F(w)=\left(\begin{array}{c}
\rho u_{g} \\
\rho u_{g}^{2}+p \\
\rho u_{g} v_{g} \\
\left(\rho e_{g}+p\right) u_{g} \\
\rho u_{p} \\
\rho u_{p}^{2} \\
\rho u_{p} v_{p} \\
\rho e_{p} u_{p} \\
N_{p} u_{p}
\end{array}\right), \\
\left(\begin{array}{c}
\rho v_{g} \\
\rho u_{g} v_{g} \\
\rho v_{g}^{2}+p \\
\left(\rho e_{g}+p\right) v_{g} \\
\rho v_{p} \\
\rho u_{p} v_{p} \\
\rho v_{p}^{2} \\
\rho e_{p} v_{p} \\
N_{p} v_{p}
\end{array}\right) .
\end{gathered}
$$

Here, the notations are classical as previously, simplifying them, $g$ and $p$ for labeling the gas and solid phase, respectively. In which, $\rho_{g}, T_{g}, u_{g}, v_{g}$, stand for density, temperature, and two-components velocity stating the single phase, $\rho_{p}, T_{p} u_{p}, v_{p}$ stand for density, temperature, and two-components velocity stating the solid 
phase. The radius $r_{p}$ is necessary to determine interactions between phases. The pressure $p$ is common for the two phases. Indeed, we impose the same pressure $p$ avoiding the contact discontinuity between gas and solid particles. These components are shared into two groups. The first one is related to equations for gas, constituted of the four first components. The second one is related to the dispersed phase, that components are from 5 to 8. The last equation solves $N_{p}$ the number of particles per unit volume. The source term $Q$ depends on heat transfer $q_{r, \theta}$ that is given by:

$$
Q(w)=\left(\begin{array}{c}
0 \\
\frac{\rho v_{g}^{2}}{r}-\rho \frac{G M}{r_{g}^{2}}+\frac{p}{r}+F_{r} \\
-\frac{\rho v_{g}}{r}+F_{\theta} \\
G M \\
-\rho u_{g} \frac{G}{r^{2}}+F_{r} u_{p}+F_{\theta} v_{p}+q_{r, \theta} \\
0 \\
\frac{\rho v_{p}^{2}}{r}-\rho \frac{G M}{r_{p}^{2}}+\frac{p}{r}-F_{r} \\
-\frac{\rho v_{p}}{r}-F_{\theta} \\
-q_{r, \theta} \\
0
\end{array}\right) .
$$

The presence of friction forces $F_{r}$ and $F_{\theta}$ in source $Q$ proves that gas and solid particles are well coupled. The eight equation model for two phase flow problems are not enough to solve the system. Indeed, we have 10 unknowns: $\rho_{g}, T_{g}, u_{g}, v_{g}, \rho_{p}, T_{p} u_{p}, v_{p}, r_{p}, p$. Two equations are necessary to close this system. The first one is obtained from the variable $N_{p}$. The second one describes the state law of a perfect gas. In the sequel, in two phase model, a small relative velocity difference is expected between gas and particles while they are couple.

\section{NUMERICAL SCHEME}

\section{A. Computational domain}

The computational domain can be subdivided into a finite number of cells. These cells cover entirely the computational domain and provide a good geometrical approximation of the flow. We studied the rotation of gas and particles around the star in a ring. This ring is confined between an inner zone of radius $r_{\text {in }}=5 \mathrm{AU}$, and an outer zone in which it is delimited by radius $r_{\text {out }}=$ 10AU. We discretize the domain on an annular grid. As a consequence, the ring is divided into $n_{r}$ and $n_{\theta}$ cells, respectively for the radial and azimuthal directions. The spatial distribution of cell is defined by the radial step $\Delta r$ and the angular step $\Delta \theta$ that given by:

$$
\left\{\begin{array}{l}
\Delta r=\frac{r_{\mathrm{out}}-r_{\mathrm{in}}}{n_{r}}, \\
\Delta \theta=\frac{2 \pi}{n_{\theta}} .
\end{array}\right.
$$

The time-space complexity of the cell is described by set of couple $\left(r_{i}, \theta_{j}\right)$, where indexes $i$ and $j$ mean theirs

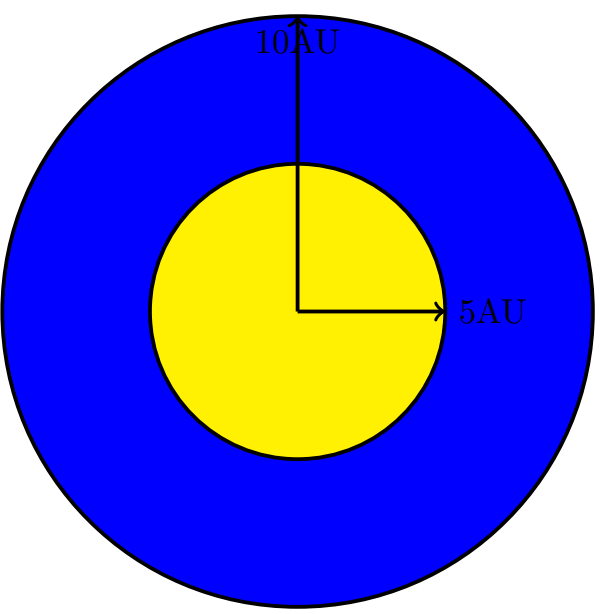

Fig. 2: Computational domain.

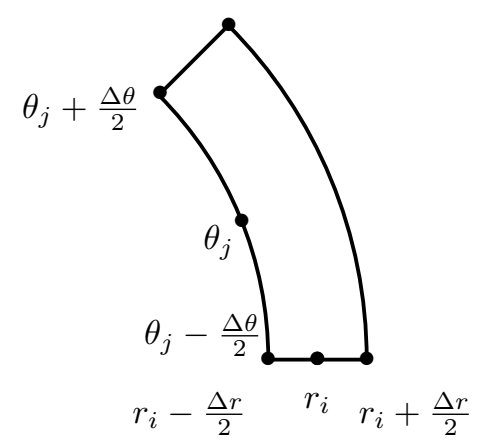

Fig. 3: Cylindrical cell.

labels according respectively to the radial and azimuthal directions. Each cell $\left(r_{i}, \theta_{j}\right)$ is configurated as follows:

$$
\left\{\begin{array}{l}
r_{i}=r_{\mathrm{in}}+\left(i-\frac{1}{2}\right) \Delta r \\
\theta_{j}=\left(j-\frac{1}{2}\right) \Delta \theta
\end{array}\right.
$$

The cell $\left(r_{i}, \theta_{j}\right)$ is illustrated in Fig. 3. This cell is bounded in the radial direction between two values $r_{i}-$ $\frac{\Delta r}{2}$ and $r_{i}+\frac{\Delta r}{2}$, while in the azimuthal direction, it varies from $\theta_{j}-\frac{\Delta \theta}{2}$ to $\theta_{j}+\frac{\Delta \theta}{2}$. The average values of the quantities are stored for each cell with using finite volume method.

The aim here is to define ghost cells, called also ghost nodes, at every point in our domain. These ghost cells are used in our method framework in order to build efficient numerical scheme which can be extended easily to multidimensional space and different time integration. These ghost cells provide the well-posed boundary conditions for the conservative equations form (11). We present them in Fig. 4 for each cell $J_{i, j}$ defined by:

$$
J_{i, j}=\left[r_{i-1 / 2}, r_{i+1 / 2}\right] \times\left[\theta_{j-1 / 2}, \theta_{j+1 / 2}\right] .
$$

The option of suitable boundary conditions at ring border stays challenging. Particularly, in the radial direction. These boundary conditions are extrapolated in order to connect with the rest of the disk. The two in- 
ner cells in the computational domain $(i=3,4)$ are extrapolated to determine the limits inside the ghost cells $(i=0,1)$. This choice is justified to provide small numerical fluctuations.

$$
\left\{\begin{array}{l}
\rho_{0, j}=\rho_{4, j}\left(\frac{r_{0}}{r_{4}}\right)^{-3 / 2}, \rho_{1, j}=\rho_{3, j}\left(\frac{r_{1}}{r_{3}}\right)^{-3 / 2}, \\
p_{0, j}=p_{4, j}\left(\frac{r_{0}}{r_{4}}\right)^{-2}, p_{1, j}=p_{3, j}\left(\frac{r_{1}}{r_{3}}\right)^{-2} .
\end{array}\right.
$$

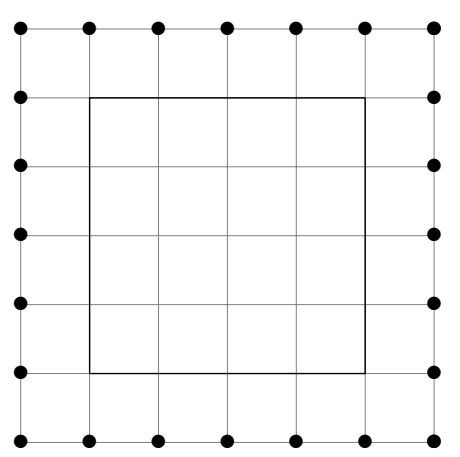

Fig. 4: Ghost cells.

Here, the number of ghost cells is exactly two, allowing to perform numerical simulations. Similarly, among the state variables, density and pressure in the outer ghost cells $\left(i=n_{r}, n_{r+1}\right)$ are obtained as well as for inner ghost cells. Besides of this, we impose periodic boundary conditions in the azimuthal direction. On others words, the variables are identical between 0 and $2 \pi$ :

$$
w(r, 0)=w(r, 2 \pi) .
$$

They are not active, which means that the physical quantities inside these cells do not evolve according to the conservation equations. Indeed, they are determined in the way to close stably the domain for the used model. Otherwise, they can also evolve over time. The principal geometrical data to be known is the surface $S_{i, j}$ defined by the angular variation:

$$
S_{i, j}=r_{i} \Delta \theta \Delta r
$$

The surface vectors allow to determine the radial and the azimuthal flux, which are related to each of the faces of the considered cell. We express the lengths of each facet presented in Fig. 5 as follows:

$$
\left\{\begin{array}{l}
\vec{l}_{i-1 / 2, j}=-r_{i-1 / 2} \Delta \theta \overrightarrow{e_{r}}, \\
\vec{l}_{i+1 / 2, j}=-r_{i+1 / 2} \Delta \theta \overrightarrow{e_{r}}, \\
\vec{l}_{i, j-1 / 2}=-\Delta r \overrightarrow{e_{\theta}}, \\
\vec{l}_{i, j+1 / 2}^{\prime}=\Delta r \overrightarrow{e_{\theta}} .
\end{array}\right.
$$

\section{B. Initial conditions}

The purpose of this subsection is to provide initial conditions that is an essential part of conceptualizing

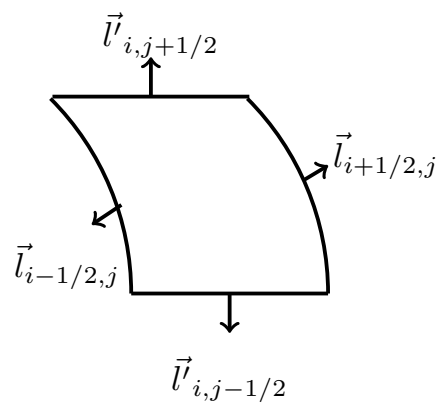

Fig. 5: The surface vectors.

and modeling the flow of particles in two phase (mixing gas and solid particles) over planetary disks. The specification of appropriate initial conditions is important in this topic because is also the part most subject to serious error by hydrologists. We explain here the concept of initial conditions and we discuss some common pitfalls in the model. In order to evolve these densities of the surface disk, we must choose an initial profile. This profile is previously introduced in [10] by Hayashi (1985). If $D_{\text {init }}$ presents one of these densities, it can be written generally in the form of a power law as follows:

$$
D_{\text {init }}=D_{0}\left(\frac{r}{r_{0}}\right)^{-d} \text {. }
$$

The density profile is calculated from the mass of planets. The amount of solid contained in the planets is distributed in rings, and then from a gas report dust. The density profile of the gas is computed by the power law (29). Otherwise, we suppose that the mixture of hydrogen molecules and helium in small quantity gives the final gas particle. These initial conditions are given as follows:

$$
\left\{\begin{array}{l}
\rho_{\text {init }}=\rho_{0}\left(\frac{r}{r_{0}}\right)^{-3 / 2}, \\
T_{\text {init }}=T_{0}\left(\frac{r}{r_{0}}\right)^{-1 / 2}, \\
p_{\text {init }}=p_{0}\left(\frac{r}{r_{0}}\right)^{-2}, \\
u_{\text {init }}=0, \\
v_{\text {init }}=\sqrt{\frac{G M}{r_{0}}\left(r_{0} / r\right)-2 \frac{p}{\rho}},
\end{array}\right.
$$

where,

$$
\left\{\begin{array}{l}
\rho_{0}=1 \cdot 4 \cdot 10^{-6} \mathrm{Kg}^{-3} \mathrm{~m}^{-3} \\
T_{0}=280 \mathrm{~K} \\
p_{0}=1.42 P a \\
r_{0}=1 A U .
\end{array}\right.
$$

C. Numerical method in one-phase

The conservation laws defined by equation in (11) related to the one-phase model, is written in following form:

$$
\partial_{t} w+\nabla \cdot f(w)=Q(w)
$$


The finite volume method is the mechanism for discretizing a partial differential equations in the formalism of compact equations. The values are computed at discrete zone on the grid. In this computational fluid dynamics package, the average of the conserved variables is stored alongside the two radial and azimuth meshes defining a control volume. In this method, volume integrals in the equation (32) containing a divergence variable, are becoming surface integrals, using the Green-Ostrogradski Theorem. Then, the terms involved in this equation are then presented as fluxes at the surfaces of each control volume. Now, we will explain why the used numerical mehod must be conservative. Indeed, the entering flux is equal to the leaving one in a given volume in each direction. Another advantage of this method is that it is clearly adjusted to allow meshing for unstructured grid. Evaluation based on the integral, rather than the differential, form of equation (11) have very wide advantages for flows that contain impacts and discontinuities [11]. Integration of equation (32) over the control volume $\Omega_{i, j}$ delimited by the surface $S_{i, j}$ gives:

$$
\int_{\Omega_{i, j}}\left(\frac{\partial w}{\partial t}+\nabla \cdot f(w)\right) d \Omega=\int_{\Omega_{i, j}} Q d \Omega .
$$

Assuming that $w_{i, j}$ is the average value on each control volume $\Omega_{i, j}$, and using Gauss's theorem, the equation (33) is written:

$$
\frac{\partial w}{\partial t} \Omega_{i, j}+\int_{\partial \Omega_{i, j}} f(w) d \Omega=\int_{\Omega_{i, j}} Q d \Omega .
$$

The conserved variable $w$, and the source term $Q$ are averaged over control volume $\Omega_{i, j}$ :

$$
\left\{\begin{aligned}
w_{i, j} & =\frac{1}{\Omega_{i, j}} \int_{\Omega_{i, j}} w d \Omega, \\
Q_{i, j} & =\frac{1}{\Omega_{i, j}} \int_{\Omega_{i, j}} Q d \Omega .
\end{aligned}\right.
$$

We start discretizing the source term on each grid $J_{i, j}$ that is approximated between two interfaces as:

$$
\int_{r_{i-\frac{1}{2}}}^{r_{i+\frac{1}{2}}} Q(w) d r=\left(\begin{array}{c}
0 \\
r_{i+1 / 2} p_{i+\frac{1}{2}}^{L}-p_{i+\frac{1}{2}}^{R} r_{i-\frac{1}{2}} \\
-\rho_{i} u_{i} v_{i} \Delta r \\
-\rho_{i} u_{i} \frac{G M}{r_{i}} \Delta r
\end{array}\right)
$$

Noticing that $p_{i}$ is a regular function depending on the variable $r$, we use an order 1 Taylor expansion of pressure term given in equality (36). Then, we obtain:

$$
r_{i+\frac{1}{2}} p_{i+\frac{1}{2}}^{L}=r_{i} p_{i}+\frac{\partial r p_{i}}{\partial r} \frac{\Delta r}{2}+\mathcal{O}\left(\Delta r^{2}\right)
$$

and,

$$
p_{i+\frac{1}{2}}^{R} r_{i-\frac{1}{2}}=r_{i} p_{i}-\frac{\partial r p_{i}}{\partial r} \frac{\Delta r}{2}+\mathcal{O}\left(\Delta r^{2}\right)
$$

The difference between these two equalities (37) and (38) gives:

$$
r_{i+\frac{1}{2}} p_{i+\frac{1}{2}}^{L}-p_{i+\frac{1}{2}}^{R} r_{i-\frac{1}{2}}=\Delta r \frac{\partial r p_{i}}{\partial r}
$$

The fluxes are computed on each of the faces, the radial flux $F$ according to the components $l$, and the azimuthal flux $G$ according to the components $l^{\prime}$. The discrete-time linear system is written in following form:

$$
\frac{\partial w_{i, j} \Omega_{i, j}}{\partial t}+\sum_{l} \vec{F} \cdot \vec{S}+\sum_{l^{\prime}} \vec{G} \cdot \vec{S}=Q_{i, j} \Omega_{i, j} .
$$

There are $N$ uniform time steps $\Delta t$. Noticing that the time step may vary at each increment time. We refer the reader to equality (52) in which it satisfies an estimate of the CFL stability condition. The initial time steps $\Delta t_{0}$ is defined as the difference between the final time $t_{f}$ when the computation is stopped and initial time $t_{0}$ when the computation begins, divided by the number $N$, i.e.

$$
\Delta t_{0}=\frac{t_{f}-t_{0}}{N}
$$

Now, coming back to integration of both sides of equality (40) with repect to variable time. The variation of quantity $w_{i, j}$ can be determined at each time step $\Delta t$ :

$$
\Delta w_{i, j}=\Delta t\left(Q_{i, j}-\frac{\sum_{l} \vec{F} \cdot \vec{S}+\sum_{l^{\prime}} \vec{G} \cdot \vec{S}}{S_{i, j}}\right) .
$$

An other discretization form of equation (42) is given by the following equality:

$$
\begin{aligned}
w_{i, j}^{n+1} & =w_{i, j}^{n}+\Delta t\left(Q_{i, j}^{n}-\frac{l_{i+\frac{1}{2}, j} F_{i+\frac{1}{2}, j}^{n}-l_{i-\frac{1}{2}, j} F_{i-\frac{1}{2}, j}^{n}}{S_{i, j}^{n}}\right) \\
& +\Delta t\left(\frac{G_{i, j+\frac{1}{2}}^{n} l_{i, j+\frac{1}{2}}^{\prime}-G_{i, j-\frac{1}{2}}^{n} l_{i, j-\frac{1}{2}}^{\prime}}{S_{i, j}^{n}}\right) .
\end{aligned}
$$

Let us explain how the flux terms involved in equality (43) are evaluated on the interface $\left(r_{i+\frac{1}{2}}, \theta_{j}\right)$ that separates two cells $J_{i-1, j}$ and $J_{i, j}$. According to the definition (28), we formally have:

$$
l_{i+\frac{1}{2}, j} F_{i+\frac{1}{2}, j}^{n}=r_{i+1 / 2} \Delta \theta \mathcal{H}\left(w_{i+\frac{1}{2}, j}^{L}, w_{i+\frac{1}{2}, j}^{R}\right) .
$$

This evaluation depends on state of the neighbors $w^{L}$ and $w^{R}$. Here, function $\mathcal{H}$ means the approximated Riemann solver, and it defines a consistent flux, i.e. $\mathcal{H}(w, w)=F(w)$. This solver takes into account the left and right states in order to interpolate variables at the interface. For instance, we will test it for the considered Godunov method. Then, the conservative vector $w$ at this interface takes the same value at center:

$$
\left\{\begin{array}{l}
w_{i+\frac{1}{2}, j}^{L}=w_{i, j}, \\
w_{i+\frac{1}{2}, j}^{R}=w_{i+1, j} .
\end{array}\right.
$$


Consequently, the discrete conservative vectors are given as:

$$
w_{i+\frac{1}{2}}^{L}=\left(\begin{array}{c}
\rho_{i} r_{i+\frac{1}{2}} \\
u_{i} \\
v_{i} r_{i+\frac{1}{2}} \\
p_{i} r_{i+\frac{1}{2}}
\end{array}\right), w_{i+\frac{1}{2}}^{R}=\left(\begin{array}{c}
\rho_{i+\frac{1}{2}} r_{i+\frac{1}{2}} \\
u_{i+1} \\
v_{i+1} r_{i+\frac{1}{2}} \\
p_{i+1} r_{i+\frac{1}{2}}
\end{array}\right)
$$

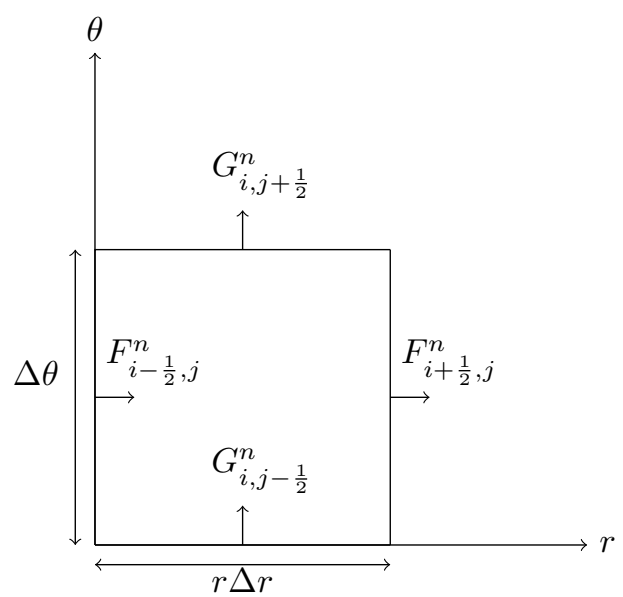

Fig. 6: Discretization by the numerical scheme over a cylindrical domain.

We categorize this method as first-order, and it is consisting in the projection of centered values. The equation defined by (11) is resolved by a splitting extrapolation method. Then, this equation (11) allows to give the following equivalent system:

$$
\left\{\begin{array}{l}
\frac{\partial w}{\partial t}+\frac{1}{r} \frac{\partial r F(w)}{\partial r}=Q(w), \\
\frac{\partial w}{\partial t}+\frac{1}{r} \frac{\partial G(w)}{\partial r}=Q(w) .
\end{array}\right.
$$

The azimuthal flux $G$ is approached by a cell-centred scheme. In order to preserve the hyperbolic character and to balance the bidimensional numerical scheme, we keep the same undimensional radial flux $F$. Assuming that $G$ is twice-derivable-function over interval $\left[r_{i}, \theta_{j}-\frac{\Delta \theta}{2}\right] \times\left[r_{i}, \theta_{j}+\frac{\Delta \theta}{2}\right]$, function $G$ is Taylor-Young expanded in $\left(r_{i}, \theta_{j}+\frac{\Delta \theta}{2}\right)$ until the order 2 to obtain:

$$
\begin{aligned}
G\left(r_{i}, \theta_{j}+\frac{\Delta \theta}{2}\right) & =G\left(r_{i}, \theta_{j}\right)+\frac{\Delta \theta}{2}\left(\frac{\partial G}{\partial \theta}\right) \\
& +\frac{(\Delta \theta)^{2}}{8}\left(\frac{\partial^{2} G}{\partial \theta^{2}}\right)+\mathcal{O}\left((\Delta \theta)^{3}\right),
\end{aligned}
$$

and function $G$ is Taylor-Young expanded in point $\left(r_{i}, \theta_{j}-\frac{\Delta \theta}{2}\right)$ until the order 2 to obtain:

$$
\begin{aligned}
G\left(r_{i}, \theta_{j}-\frac{\Delta \theta}{2}\right) & =G\left(r_{i}, \theta_{j}\right)-\frac{\Delta \theta}{2}\left(\frac{\partial G}{\partial \theta}\right) \\
& +\frac{(\Delta \theta)^{2}}{8}\left(\frac{\partial^{2} G}{\partial \theta^{2}}\right)+\mathcal{O}\left((\Delta \theta)^{3}\right)
\end{aligned}
$$

Subtracting equation (48) from equation (49), we get:

$$
\frac{G_{i, j+\frac{1}{2}}-G_{i, j-\frac{1}{2}}}{\Delta \theta}=\frac{\partial G}{\partial \theta}+\mathcal{O}\left((\Delta \theta)^{2}\right) .
$$

The second-order discretization for the flux $G$ is defined from the upstream approximation at the interface $\left(r_{i}, \theta_{j+\frac{1}{2}}\right)$ and the downstream approximation at the interface $\left(r_{i}, \theta_{j-\frac{1}{2}}\right)$ as follows:

$$
G_{i, j+\frac{1}{2}}=\left(\begin{array}{c}
\rho_{i, j+\frac{1}{2}} v_{i, j+\frac{1}{2}} \\
\rho_{i, j+\frac{1}{2}} u_{i, j+\frac{1}{2}} v_{i, j+\frac{1}{2}} \\
\rho_{i, j+\frac{1}{2}} v_{i, j+\frac{1}{2}}^{2}+p_{i, j+\frac{1}{2}} \\
\left(\rho_{i, j+\frac{1}{2}} e_{i, j+\frac{1}{2}}+p_{i, j+\frac{1}{2}}\right) v_{i, j+\frac{1}{2}}
\end{array}\right) .
$$

Insisting here that first-order well-balanced scheme is based on constructing a piecewise constant way for the density and velocity, in which the associated cells are averaged. Next, the pressure is deduced with hydrostatic equilibrium. This contributes for the discretization numerically of the radial flux $F$. This flux is fused directly with the azimutal one $G$ which is computed by equality (51).

The Courant-Friedrichs-Lewy is a dimensionless quantity, which is denoted shortly CFL for meaning a fundamental restriction. It leads to the numerical solution defined by the discretization process for a quickly convergence. This number is involved in the numerical analysis in order to maintain a stability of the solution, when if it existes. Considering the Euler equations (11) in one-phase which is presented by a compressible gas. In the low Mach number regimes, the acoustic waves influence the capturing the motion of this fluid. On the other hand, they dont not impact loss of energy that is stays negligible. In order to affect the efficiency of the numerical method itself, the time step is based on wavespeeds at cell centers. If the cells are too small compared with the time step, numerical smearing may occur leading to instability and in some cases divergence. Since time step $\Delta t \leq \Delta t_{0}$ depends on chosen cell $\Omega_{i, j}$, then it is given by:

$$
\Delta t=\frac{C_{\mathrm{CFL}}}{\frac{\max _{i, j}\left|u_{i, j}+c_{i, j}^{r, \theta, n}\right|}{r_{i+1 / 2} \Delta r}+\frac{\max _{i, j}\left|v_{i, j}+c_{i, j}^{r, \theta, n}\right|}{\Delta \theta}},
$$

where $c_{i, j}^{r, \theta, n}$ is the speed of propagation inside the cell according to the radial and azimuthal directions. This velocity in the two-dimensional case is defined as:

$$
\forall 0 \leq i \leq n_{r}, 0 \leq j \leq n_{\theta}, c_{i, j}^{r, \theta, n}=\sqrt{\frac{\gamma p_{i, j}}{\rho_{i, j}}}
$$

The coefficient CFL is chosen from such so that the condition of numerical stability is verified. The flux depend necessarily on coefficient CFL, for the method of $1^{\text {st }}$ order, we take in practice $C_{\mathrm{CFL}} \simeq 0.9$. In what follows, we realize one test that consists in following the behavior of 
the disc in the absence of any perturbations. The goal of this test is to verify the stability of the flow. For that, comparing the initial profile of each variable denoted var (stands for density, pressure, temperature, etc) to the obtained one by simulation with using a large number of integration time. In the sequel, we only focus this comparison on the radial direction. That why each variable is averaged following the azimuthal direction under this form:

$$
\operatorname{var} \mapsto \frac{1}{n_{\theta}} \sum_{i=1}^{n_{\theta}} \operatorname{var}\left(r, \theta_{i}\right)
$$

Comparing the approached azimuthal velocity generated by the developed numerical code to its explicit expression given by equality (18). We present these velocities in the Fig. 7. The blue and red diagrams present the exact azimuthal velocity and its approximation, respectively. This simulation shows that the gas phase does not give much satisfaction due to bad preservation of the stationary solution. In order to overcome these important effects, we need to limit the time computation and refine mesh. There is an other reason for this poor conservation. Indeed, the radial velocity does not converge to the initial zero value. This appears clearly in the Fig. 8. The oscillatory precision appears, near from $10^{-11}$, meaning of something physically happens. It is concluded that the numerical scheme does not behave correctly.

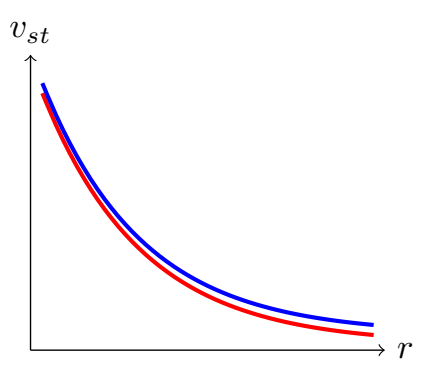

Fig. 7: Azimuthal velocity $v_{s t}$.

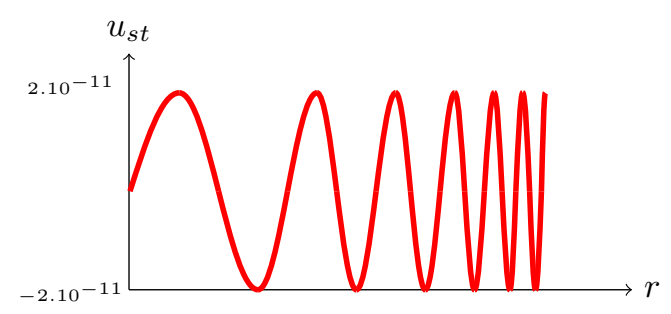

Fig. 8: Radial approached velocity $u_{s t}$.

In order to show the applicability and performance of the proposed well-balanced scheme. For comparison, we present in Fig. 9 the radial velocity result obtained with a standard (unbalanced) base scheme. It shows that the scheme does not react well since the model reacts to perturbations and produces significant oscillations. On other hand, as one can see in Fig. 10, the well-balanced scheme correctly captures the behavior of the solution. Indeed, the geometry of the domain does not influence the nature of the azimuthal velocity for relaxed and refined meshes.

Continuing here to perform a resolution test with giving convergence results for the stated spatial resolution. It is known that numerical simulations of one-phase flow are always far to be predictive, particularly when considering system of Euler equations. Many reasons should be responsible for this problem, inducted by mesh quality, numerical scheme, etc. In order to give an answer to this problem, we should aim to state an estimate for the relative error in some physical variable. This error states perturbation analysis in density, pressure and internal energy shown in Fig. 11 which is interpreted as follows. The system is stable, with very low relative error.

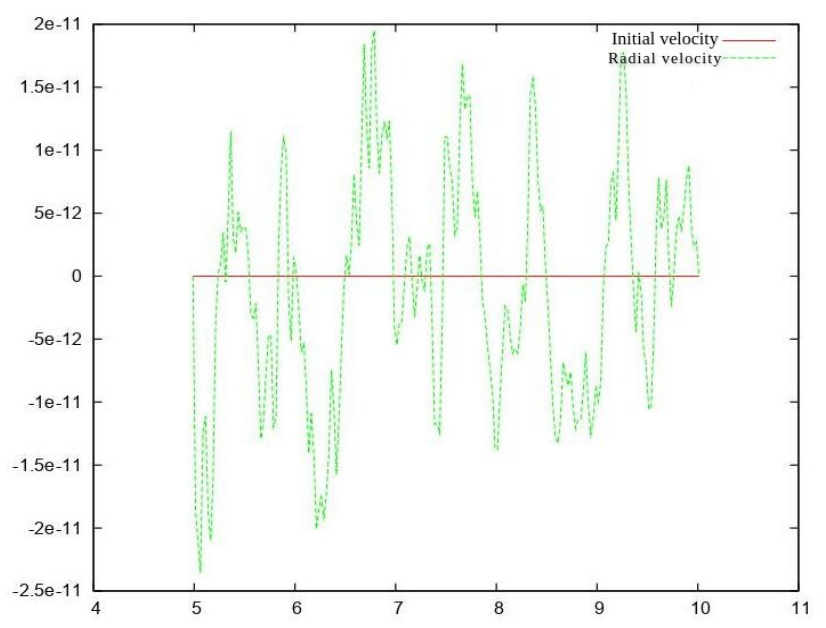

Fig. 9: Exact and approached radial velocities for the standard numerical scheme.
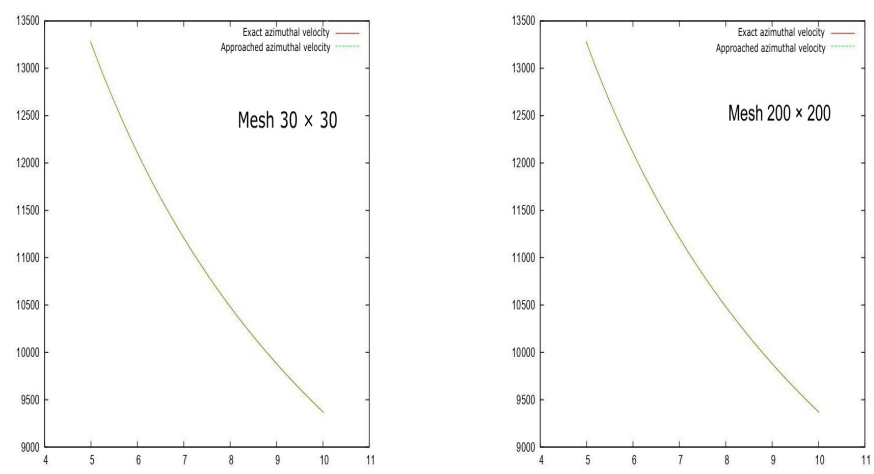

Fig. 10: Exact and approached azimuthal velocities between coarse mesh, i.e. $\left(n_{r}, n_{\theta}\right)=(30,30)$, and refined mesh, i.e. $\left(n_{r}, n_{\theta}\right)=(200,200)$ for well-balanced scheme. 

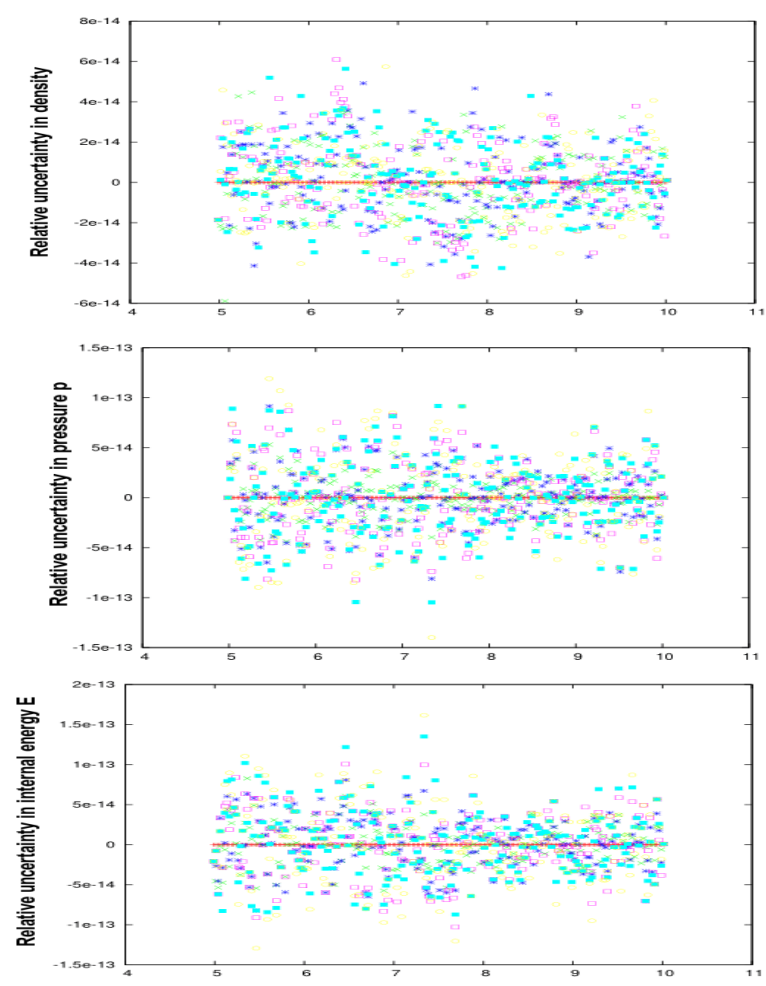

Fig. 11: Relative uncertainty for three quantities. The first one is the density $\rho$ presented in left diagram, the second one is the pressure $p$ presented in middle diagram, the last one is the internal energy $E$ shown in right diagram.

\section{TESTING THE CODE ACCURACY IN TWO-DIMENSIONAL SPACE}

We test in this section the ability of the code to conserve the disk in steady-state. Indeed, the rotation of gas density around the central star stays the same over time. Here, we show the results that regime steady-state determine the solution in different time durations. This solution is unchanging in continuous instants.
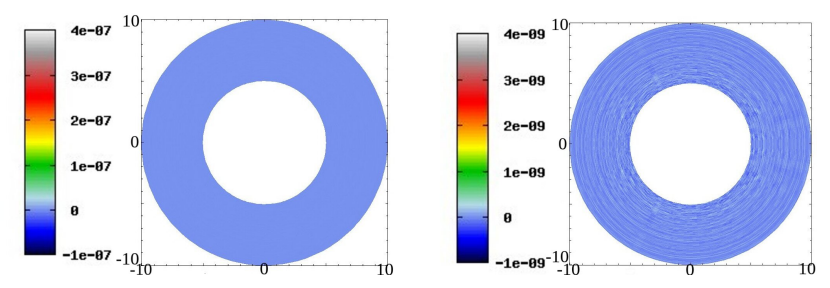

Fig. 12: Disk at initial Fig. 13: Disk at initial time, with scale $10^{-7}$.

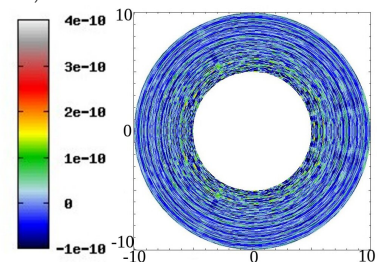
time, with scale $10^{-10}$.

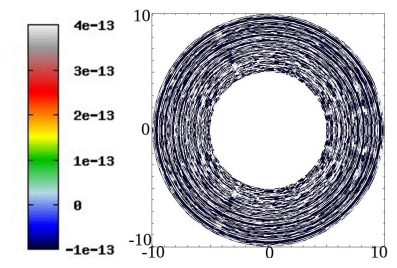

Fig. 14: Disk after 500 Fig. 15: Disk after 1000 years, with scale $10^{-10}$. years, with scale $10^{-14}$.
In these simulations, we keep the same value of inner and outer radii, introduced previously in subsection A.. These results are performed over 1000 years for twodimensional space. A reasonable grid resolution are 100 cells in two space directions, i.e. $n_{r}=n_{\theta}=100$. Now, we are checking whether the code has the necessary accuracy to calculate the stationary solutions that remain uniform over time. The $2 \mathrm{D}$ evolution of the density $\rho$ is presented in Figs. 12, 13, 14 and 15. Fig. 12 presents that there is no fluctuations with a scale of approximation $10^{-7}$ of density $\rho$. However on a smaller scale than $10^{-7}$, the disturbances presented in Figs. 13, 14 and 15 begin to appear on the disk.

\section{Stability AND LONG-TERM EVOLUTION}

This section adresses to study the perturbation of the vortices in the disk. In this study, we are neglecting the various physical effects such as turbulence. Testing here the evolution of the disc, with adding an overdensity and an overpressure to the stationary solution. The target is to study how the disc reacts with the presence of overdensity. The variables involved in this model are defined by:

- $(X, Y)$ : the cartesian reference in rotation linked to the vortex;

- $(x, y)$ : the inertial cartesian coordinates system;

- $R=\sqrt{X^{2}+Y^{2}}$ : the distance to the center of the vortex;

- $(r, \theta)$ : the inertial cylindrical coordinate system;

- $\left(x_{0}, y_{0}\right)$ : the coordinates of the vortex center in the reference.

We recall that $r$ is the distance from the sun to the vortex center expressed in astronomical unit. As shown in Fig. 16, the change reference for returning to the initial cylindrical system $(r, \theta)$ is:

$$
\left\{\begin{array}{l}
X=r \cos \theta-x_{0} \\
Y=r \sin \theta-y_{0} \\
R^{2}=r^{2}+x_{0}^{2}+y_{0}^{2}-2 r\left(x_{0} \cos \theta+y_{0} \sin \theta\right) .
\end{array}\right.
$$

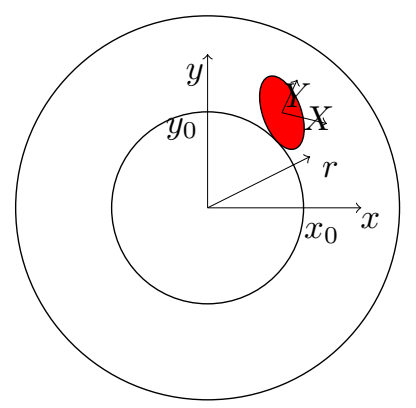

Fig. 16: Different coordinate system. 
The perturbation of the velocity in the reference $(r, \theta)$ is written:

$$
\left\{\begin{array}{l}
\Delta V_{r}=\cos \theta \Delta V_{X}+\sin \theta \Delta V_{Y}, \\
\Delta V_{\theta}=\cos \theta \Delta V_{Y}-\sin \theta \Delta V_{X} .
\end{array}\right.
$$

On other hand, the perturbation of the velocity in the cartesian coordinate system linked to the vortex is written:

$$
\left\{\begin{array}{l}
\Delta V_{X}=\cos \theta \Delta V_{r}+\sin \theta \Delta V_{\theta}, \\
\Delta V_{Y}=\cos \theta \Delta V_{\theta}-\sin \theta \Delta V_{r} .
\end{array}\right.
$$

It is convenient to use this vortex described above, because it will be simply implemented. On other word, it does not require a mesh refinement. Noticing that a Gaussian overdensity is not necessarily a vortex, because a vortex turns around itself. On the contrary, overdensity does not. In order to test the stability of the disc, we will add the Gaussian perturbations, which are randomly distributed inside the disc.
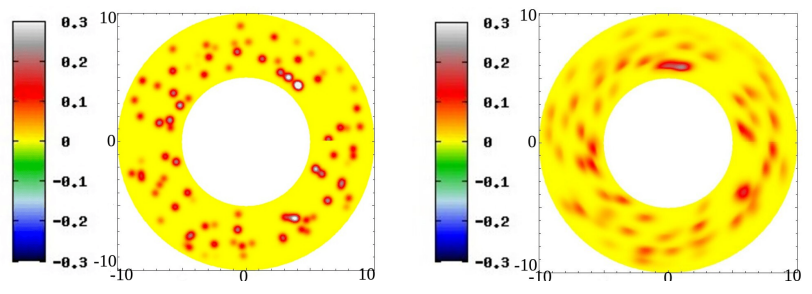

Fig. 17: Disk at initial Fig. 18: Disk after 10 time.

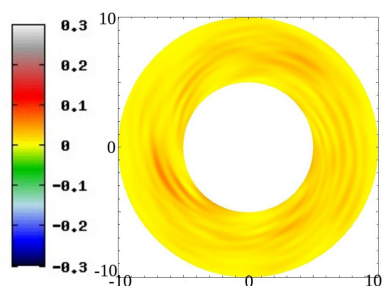
years.

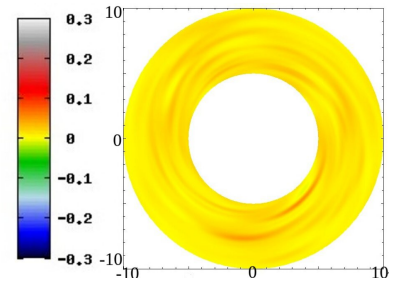

Fig. 19: Disk after 15 Fig. 20: Disk after 25 years. years.

The average width of this Gaussian function takes the value $0.075 \mathrm{AU}$. It means that a small amplitude of noise is considered. We assure that a set of vortices stays always in the disk. We are interested to follow them in order to know how much time they are survived. Seeing later that they will be deformed, and disappearing after only 3 rotations. For our numerical setup, a refine mesh is used to get more stability precision, the grid size is $200 \times 200$, i.e. $n_{r}=n_{\theta}=200$.

The stability of Rossby waves has been discussed by authors Lovelace and Li [12, 13] from 1999 to 2001 in the evolution of protoplanetary disks. In [12], the authors investigate the stability problem of non-magnetized Keplerian disks. They study particularly a linear instability of non-axisymmetric perturbations for conditions where the disk variables, such as pressure density has saturated radial gradients. On other hand, the nonlinear instability has been studied by the authors in [13]. They consid- ered thin disks. The numerical results showing this wave instability are the revelation for many scientists, precisely in meteorological slant. This instability makes loss the atmospheric prevision which existes in some models. This energy faillure is due to for the presence of the light, contributing to the interactions between particles.

In what follows, we try to produce one of the linear Rossby stability, that why we use Gaussian bump. Figs. 17, 18, 19 and 20 show that different evolutions of the gas density $\rho$ in disk. We present in Fig. 17, the initial state of Gaussian bump distributed on disk. Inside the vortex, the density field is given to get a Gaussian density like the background-disk case. The vortex survived more than 3 rotations and its structure was quasi-steady with only two days duration meaning source of numerical diffusion.

\section{THREE-DIMENSIONAL FINITE VOLUME METHOD}

We will extend the $2 \mathrm{D}$ numerical code for the simulation to three dimensional space by taking into account the height in the z-axis direction. The success to build the numerical scheme in bidimensional space allows and ensures to be expanded to three dimensional one. The aim of this work is to keep the stationary solutions with a good precision. Consequently, we consider the physical domain to be a toroid around the Sun. It is sufficient to add another dimension of space, relative to the two-dimensional scheme. The radial and azimuthal discretizations will remain the same. However, the following $\mathrm{z}$-direction is discretized between two opposite values $-H$ and $H$, as it is shown in Fig. 21.

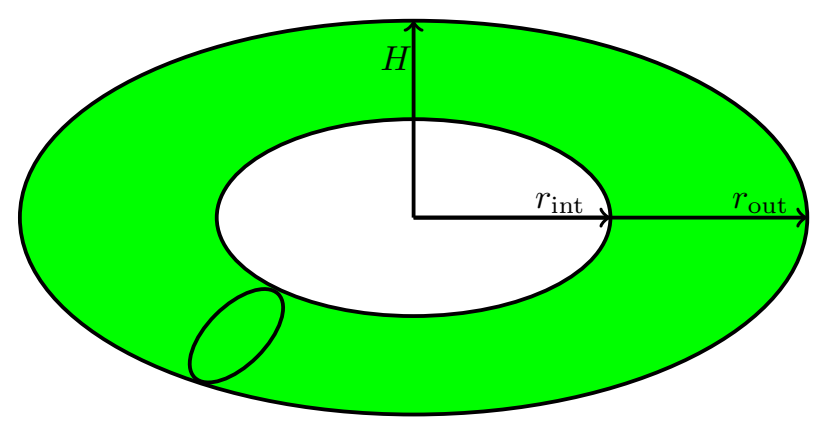

Fig. 21: Computational domain in 3D.

The system of Euler equations given by (11) is extended from bidimensional space to three-dimensional one in cylindrical coordinates as:

$$
\frac{\partial w}{\partial t}+\frac{1}{r} \frac{\partial r F(w)}{\partial r}+\frac{1}{r} \frac{\partial G(w)}{\partial \theta}+\frac{1}{r} \frac{\partial H(w)}{\partial z}=Q(w)
$$

The configuration of the cell labeled in our threedimensional grid around the star is illustrated in Fig. VII.. Since the cell is labeled $(i, j, k)$ with the index $k$ in the $\mathrm{z}$-direction, its position is given by:

$$
\left\{\begin{array}{l}
r_{i}=r_{\text {in }}+\left(i-\frac{1}{2}\right) \Delta r \\
\theta_{j}=\left(j-\frac{1}{2}\right) \Delta \theta \\
z_{k}=\left(k-\frac{1}{2}\right) \Delta z
\end{array}\right.
$$




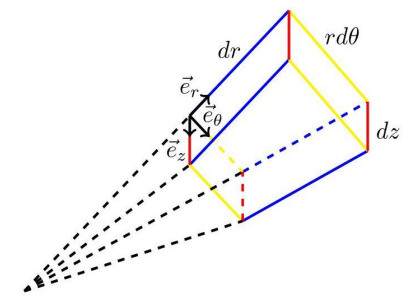

Fig. 22: Cylindrical coordinates $\left(r_{i}, \theta_{j}, z_{k}\right)$ in $3 \mathrm{D}$.

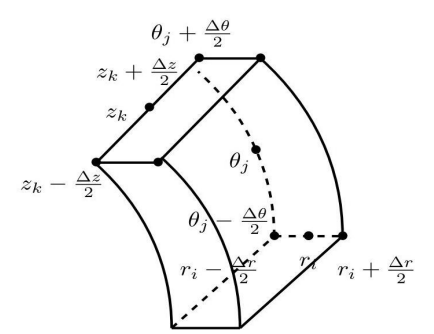

Fig. 23: Cylindrical cell in 3D.

The discrete-time linear system of above equation is done as in two-dimensional space (see equality (42)),

$$
\Delta w_{i, j, k}=\Delta t\left(Q_{i, j, k}-\frac{\sum_{l} \vec{F} \cdot \vec{S}+\sum_{l^{\prime}} \vec{G} \cdot \vec{S}+\sum_{l^{\prime \prime}} \vec{H} \cdot \vec{S}}{V_{i, j, k}}\right) .
$$

where $l$ " means the length of each facet in the z-direction, i.e. $\vec{l}_{i, j, k-1 / 2}=-\Delta z \vec{e}_{z}, \vec{l}_{i, j, k+1 / 2}=\Delta z \overrightarrow{e_{z}}$, and control volume of each cell denoted by $V_{i, j, k}$, defined as $V_{i, j, k}=r_{i} \Delta \theta \Delta r \Delta z$. The discrete formalism of equation (57) involving the three indices $i, j$ and $k$ going through in the height direction is described as follows:

$$
\begin{aligned}
w_{i, j, k}^{n+1} & =w_{i, j, k}^{n}+\Delta t Q_{i, j, k}^{n} \\
& +\Delta t\left(-\frac{l_{i+\frac{1}{2}, j, k} F_{i+\frac{1}{2}, j, k}^{n}-l_{i-\frac{1}{2}, j, k} F_{i-\frac{1}{2}, j, k}^{n}}{V_{i, j, k}^{n}}\right) . \\
& +\Delta t\left(\frac{G_{i, j+\frac{1}{2}, k}^{n} l_{i, j+\frac{1}{2}, k}^{\prime}-G_{i, j-\frac{1}{2}, k}^{n} l_{i, j-\frac{1}{2}, k}^{\prime}}{V_{i, j, k}^{n}}\right) \\
& +\Delta t\left(\frac{H_{i, j, k+\frac{1}{2}}^{n} l_{i, j, k+\frac{1}{2}}^{\prime}-H_{i, j, k-\frac{1}{2}}^{n} l^{\prime \prime}{ }_{i, j, k-\frac{1}{2}}}{V_{i, j, k}^{n}}\right) .
\end{aligned}
$$

This preliminary study shows that the development of the 3D numerical code should not present a major problem. This numerical scheme is based on the Riemann solver problem at each interface, by determining the approached solution from left and right states. Here, we used the centered fluxes into the azimuthal and height directions in order to balance the scheme.

\section{CONCLUSIONS}

The proposed work is set in the protoplanetary nebula before coupling solid and gas phases. This work involves constructing the $2 \mathrm{D}$ numerical code for the planetisimal formation. In summary, we have applied the lifetime of gaz phase in a protoplanetary disk. Besides, we have presented a specific method to construct wellbalanced scheme for the conservative Euler system. This basic study programs the development of the 3D numerical code with balancing the fluxes. The new numerical scheme allows to better represent the steady Keplerian flow. This accuracy is showed by the long term integrations. The future part of the project to explore is summarized by following stages:

- We will develop in a second-order well-balanced scheme for the compressible Euler equations with keeping the gravitational source term. This extension from first-order to the second one relies on a pressure reconstruction with underlying hydrostatic equilibrium. It allows many advances for a highlyaccurate numerical solutions.

- Numerical tests will be performed by comparing the accuracy and efficiency of the new multi-slopes methods with the first order method.

\section{ACKNOWLEDGMENT}

This work was supported by the National Center for Scientific Research. I would like to thank the anonymous reviewers for their helpful comments and valuable suggestions to improve this article.

\section{REFERENCES}

[1] S. Udry, D. Fischer, and D. Queloz, "Protostars and planets v," Reipurth, D. Jewitt, K. Keil, eds, pp. 685-699, 2007

[2] Z. Wang, D. Chakrabarty, and D. L. Kaplan, "A debris disk around an isolated young neutron star," Nature, vol. 440, no. 7085, pp. 772-775, 2006.

[3] P. Artymowicz and S. H. Lubow, "Dynamics of binary-disk interaction. 1: gap sizes," apj, vol. 421, pp. 651-667, Feb. 1994.

[4] A. Wolszczan and D. A. Frail, "A planetary system around the millisecond pulsar psr1257+ 12," Nature, vol. 355, no. 6356, pp. 145-147, 1992.

[5] T. Chakkour, "Simulations numriques des tubes avec contraction brusque sur openfoam," Thermodynamique des interfaces et mcanique des fluides, vol. 1, no. 1, 2017.

[6] M. Ishii, "Thermo-fluid dynamic theory of two-phase flow., volume 22 of direction des tudes et recherches lectricit de france," Eyrolles, Paris, 1975.

[7] A. Kapila, R. Menikoff, J. Bdzil, S. Son, and D. S. Stewart, "Two-phase modeling of deflagration-todetonation transition in granular materials: Reduced equations," Physics of fluids, vol. 13, no. 10, pp. 30023024, 2001.

[8] R. Saurel and R. Abgrall, "A multiphase godunov method for compressible multifluid and multiphase 
flows," Journal of Computational Physics, vol. 150, no. 2, pp. 425-467, 1999.

[9] A. Murrone and H. Guillard, "A five equation reduced model for compressible two phase flow problems," Journal of Computational Physics, vol. 202, no. 2, pp. 664-698, 2005.

[10] C. Hayashi, K. Nakazawa, and Y. Nakagawa, "Formation of the solar system," in Protostars and planets II, pp. 1100-1153, 1985.

[11] R. J. LeVeque, "Finite-volume methods for nonlinear elasticity in heterogeneous media," International Journal for Numerical Methods in Fluids, vol. 40, no. 1-2, pp. 93-104, 2002.

[12] R. Lovelace, H. Li, S. Colgate, and A. Nelson, "Rossby wave instability of keplerian accretion disks," The Astrophysical Journal, vol. 513, no. 2, p. $805,1999$.

[13] H. Li, S. Colgate, B. Wendroff, and R. Liska, "Rossby wave instability of thin accretion disks. iii. nonlinear simulations," The Astrophysical Journal, vol. 551, no. 2, p. 874, 2001.

[14] T. Chakkour, "Some notes about the continuous-intime financial model," Abstract And Applied Analysis, vol. $17,3 / 17$.

[15] T. Chakkour, "Inverse problem stability of a continuous-in-time financial model," Acta Mathematica Scientia, vol. 39, no. 5, pp. 1423-1439, 7/19.

[16] T. Chakkour, "Implementing some mathematical operators for a continuous-in-time financial model," Engineering Mathematics Letters, vol. 17, no. 2, pp. 1-14, 2/17.

[17] R. Nakatani, T. Hosokawa, N. Yoshida, H. Nomura, R. Kuiper, "Radiation hydrodynamics simulations of photoevaporation of protoplanetary disks by ultraviolet radiation: Metallicity dependence," The Astrophysical Journal, vol. 857, no. 1, p.57, 2018.

[18] T. Chakkour, F. Benkhaldoun, "Slurry Pipeline for fluid transients in pressurized conduits," International Journal of Mechanics, vol. 14, 2020.

[19] P. Sarma, P. K. Karmakar, "Nonlinear dynamics of structure formation in protoplanetary disks," Chinese Journal of Physics, vol. 74, pp. 9-19, 2021.

[20] T. Chakkour, "Reengineering of aerodynamic flowfield module," Engineering Mathematics Letters. Article ID 4, 2018: http-scik.

[21] T. Watanabe, "Numerical Simulation of Droplet Combustion using Volume-of-Fluid Method," WSEAS Transactions on Heat and Mass Transfer, vol. 14, pp. 38-44, 2019 .

[22] M. Y. SIKKANDAR, N. M. SUDHARSAN, S. BEGUM, E. NG, "Computational Fluid Dynamics: A Technique to Solve Complex Biomedical Engineering Problems-A Review," WSEAS Transactions on Biology and Biomedicine, vol. 16, pp. 121-137, 2019.

[23] R. Käppeli, S. Mishra, "A well-balanced finite volume scheme for the euler equations with gravitationthe exact preservation of hydrostatic equilibrium with arbitrary entropy stratification," Astronomy \& Astrophysics, vol. 587, pp. A94, 2016.
[24] O. Gressel, J. P. Ramsey, C. Brinch, R. P. Nelson, N. J. Turner, S. Bruderer, "Global hydromagnetic simulations of protoplanetary disks with stellar irradiation and simplified thermochemistry," The Astrophysical Journal, vol. 896, no. 2, p.126, 2020.

[25] R. T. Tominaga, S. I. Inutsuka, S. Z. Takahashi, "Non-linear development of secular gravitational instability in protoplanetary disks," Publications of the Astronomical Society of Japan, vol. 70, no. 1, 2018.

[26] R. Li, A. N. Youdin, J. B. Simon, "On the numerical robustness of the streaming instability: Particle concentration and gas dynamics in protoplanetary disks," The Astrophysical Journal, vol. 862, no. 1, 2018.

[27] T. G Elizarova, , A. A. Zlotnik, M. A. Istomina. "Hydrodynamical aspects of the formation of spiralvortical structures in rotating gaseous disks," Astronomy Reports, vol. 62, no. 1, pp. 9-18, 2018.

[28] P. Bentez-Llambay, L. Krapp, M. E. Pessah, "Asymptotically Stable Numerical Method for Multispecies Momentum Transfer: Gas and Multifluid Dust Test Suite and Implementation in FARGO3D," The Astrophysical Journal Supplement Series, vol. 241, no. 2, p.25, 2019.

[29] L. Grosheintz-Laval, R. Käppeli, "High-order wellbalanced finite volume schemes for the Euler equations with gravitation," Journal of Computational Physics, vol. 378, pp. 324-343, 2019.

[30] G. Li, Y. Xing, "Well-balanced discontinuous Galerkin methods with hydrostatic reconstruction for the Euler equations with gravitation," Journal of Computational Physics, vol. 352, pp. 445-462, 2018.

[31] A. Chertock, S. Cui, A. Kurganov, S. N. Özcan, E. Tadmor, "Well-balanced schemes for the Euler equations with gravitation: Conservative formulation using global fluxes," Journal of Computational Physics, vol. 358, pp. 36-52, 2018.

[32] B. Xie, X. Deng, Z. Sun, F. Xiao, "A hybrid pressure-density-based mach uniform algorithm for $2 \mathrm{~d}$ euler equations on unstructured grids by using multi-moment finite volume method," Journal of Computational Physics, vol. 335, pp. 637-663, 2017.

[33] K. Carlberg, Y. Choi, S. Sargsyan, "Conservative model reduction for finite-volume models," Journal of Computational Physics, vol. 371, pp. 280-314, 2018.

[34] T. Chakkour, E. Frenod, "Inverse problem and concentration method of a continuous-in-time financial model," International Journal of Financial Engineering, vol. 3, no. 2, pp. 1650016, 2/16.

[35] D. S. Balsara, "Higher-order accurate space-time schemes for computational astrophysicsPart I: finite volume methods," Living reviews in computational astrophysics, vol. 3, no. 1, pp. 1-138, 2017.

\section{Creative Commons Attribution License 4.0 (At-} tribution 4.0 International, CC BY 4.0)

This article is published under the terms of the Creative Commons Attribution License 4.0

https://creativecommons.org/licenses/by/4.0/deed.en_US 